\title{
Los sitios arqueológicos de Chilón, Nachoj, Mukana y Natentsun, MUNICIPIO DE ChILÓN, ChIAPAS
}

\author{
Chilon, Nachoj, Mukana and Natentsun Archaeological Sites, Municipal Area of Chilon, Chiapas
}

\author{
Alejandro Sheseña-Hernández \\ Alejandro Tovalín-Ahumada
}

Resumen: Los sitios arqueológicos de Chilón, Mukana, Natentsun y Nachoj, localizados en el municipio de Chilón, al norte de Chiapas, representan un complejo histórico de relevancia por sus restos arquitectónicos y escultóricos. Dado que las pocas referencias que existen sobre estos monumentos se encuentran olvidadas y dispersas, e incluso inéditas, una buena manera de retomar la discusión sobre estos restos arqueológicos para la discusión actual es reuniendo y sistematizando la información existente para, a continuación, ensayar una nueva valoración histórica sobre ellos a la luz de las últimas ideas sobre la historia de los antiguos mayas.

Palabras clave: antiguos mayas, sitios arqueológicos.

Abstracts: Since there are few references on Chilon, Mukana, Nachoj and Natentsun archaeological sites, located in the municipality of Chilon, Chiapas, representing themselves for their architectural and sculptural remains a relevance historical complex, are neglected and dispersed, a good way to rescue these monuments to the current discussion is collecting and systematizing the existing information, then try a new historical appraisal of them in light of the latest ideas managed on the history of the ancient Maya.

Keywords: ancient Maya, archaeological sites.

Alejandro Sheseña Hernández, doctor en historia por la Universidad Estatal de Voronezh, Rusia. Investigador de tiempo completo adscrito a la Dirección General de Investigaciones de la Universidad Veracruzana, México. Correo electrónico: sesena@hotmail.com.

Alejandro Tovalín Ahumada, maestro en historia por la Universidad Autónoma de Chiapas y la Universidad de Ciencias y Artes de Chiapas, México. Investigador del Centro INAH-Chiapas, México. Correo electrónico: ftovalin@hotmail.com.
Enviado a dictamen: 15 de marzo de 2013.

Aprobación: 20 de abril de 2013.

Revisiones: 1. 


\section{Introducción}

A proximadamente a un kilómetro en línea recta al suroeste del actual poblado de Chilón, Chiapas, en la cima de una pequeña cordillera dispuesta al poniente del poblado y que corre en un eje noroestesureste, se levantan cuatro crestas - 1100 metros sobre el nivel del mar- que, por la forma y cercanía entre ellas, constituyen un singular conjunto que puede distinguirse fácilmente desde el poblado (ver fotografía panorámica en Becquelin y Baudez, 1982: 1231). Cada una de estas cuatro crestas presenta en su cima, entre pinos y robles, restos de varias estructuras prehispánicas, bastante dañadas por el saqueo, pertenecientes todas al periodo Clásico Tardío (ver mapa 1). Por la escasa distancia que media entre los restos, es claro que los cuatro sitios conforman un complejo arqueológico único. Uno de ellos conservaba bellos restos de estuco modelado, lo que indica, junto con la cantidad y extensión de las estructuras presentes, que este complejo poseía sin duda determinada relevancia en el pasado prehispánico de la región de Chilón. ${ }^{1}$

Dado que las pocas referencias que existen sobre este conjunto de sitios arqueológicos se encuentran olvidadas y dispersas, e incluso inéditas, una buena manera de rescatar estos monumentos para la discusión actual tal vez sea reuniendo y sistematizando la información existente para, a continuación, ensayar una nueva valoración histórica sobre ellos a la luz de las últimas ideas manejadas sobre la historia de los antiguos mayas. El presente artículo tiene como fin abordar estas dos tareas.

\section{Nombres y distribución de los sitios}

Estos cuatro sitios arqueológicos, muy semejantes por su estilo a Toniná, son conocidos como Chilón -nombre tomado del poblado actual homónimo, ubicado en el extremo norte del eje-, Mukana — "Casa Enterrada", contiguo al anterior hacia el sur-, Nachoj - "Casa del Jaguar", el último en el extremo sur del eje- y Natentsun - "Casa del Chivo", el sitio ubicado entre los dos últimos; NTZ en la nomenclatura de
Berthold Riese, 2004-. El nombre de este último, "Casa del Chivo", se lo habrían otorgado los indígenas locales debido a la presunta existencia de la pintura roja de un chivo en el interior de una de las estructuras; de hecho, los lugareños habrían considerado que el sitio era la morada de cierta divinidad con forma de chivo a la cual rendían allí ofrendas periódicamente (Cordan, 1959: 78). Actualmente todos los sitios se encuentran en terrenos de distintas organizaciones ejidales indígenas.

\section{Historia de las investigaciones}

Las primeras noticias acerca de la existencia de Natentsun -y de sus vecinos Chilón, Nachoj y Mukana-las tuvo Wolfgang Cordan (1959). Advertido por un conocido suyo sobre el reciente descubrimiento - y saqueo- de estos sitios arqueológicos, Cordan decidió visitarlos en 1958. Los monumentos habían sido descubiertos durante los trabajos de trazo de las colindancias del poblado de Chilón (Adolfo Vera, comunicación personal, 2010). Todo parece indicar que fue el entonces dueño del terreno, Alfonso Jiménez, el primero que tuvo conocimiento de los restos arqueológicos y el que inició el saqueo.

Cordan no tuvo éxito en encontrar la mencionada pintura del chivo que, según los lugareños, se conservaba en el interior de una de las estructuras de Natentsun - pues fue destruida por saqueadores posteriores-. Sin embargo, sobre uno de los muros de esta misma edificación descubrió la bella cabeza de un ser fantástico de estilo Clásico Tardío que, elaborada en estuco, parecía haber formado parte de un relieve mucho más grande recientemente destruido. Esta cabeza, que todavía se encontraba fijada al muro, afortunadamente fue fotografiada por Cordan al instante, pues más tarde sería destruida (Cordan, 1959) (ver foto 1).

Ante la presión de Cordan, Jiménez accedió a mostrar otro fragmento de este relieve que él mismo había despegado y sustraído. Se trataba de otra hermosa escultura de estuco del mismo período que mostraba la cabeza de otro ser sobrenatural. Esta segunda pieza, junto con otras menores contemporáneas, también extraídas de Natentsun, se encontraba oculta 
justamente en la casa de Jiménez (Cordan, 1959: 70-113). Después de que Cordan fotografiara las piezas, todas ellas fueron retiradas de la casa de Jiménez y enviadas a Yajalón, en donde fueron de nuevo fotografiadas por Walter Sellschopp (ver fotos 2 y 3). Posteriormente, el señor José Óscar Moscoso, entonces recaudador de Hacienda Federal en Yajalón, las empacó para su envío al museo del Instituto Nacional de Antropología e Historia (INAH) en Tuxtla Gutiérrez, Chiapas (Augusto Sellschopp, comunicación personal 2010).

Frans Blom y Gertrude Duby visitaron Natentsun poco después, en octubre de 1958. Duby todavía logró tomar fotografías de la cabeza de estuco fijada al muro antes de su destrucción (ver foto 4). Con estas fotografías, Blom y Duby elaboraron un reporte que fue enviado al INAH para su atención (Blom y Duby, 1958). Más tarde, Blom volvería a insistir en la necesidad de realizar investigaciones arqueológicas en el lugar (Blom, 1961: 118; 2004: 116). Para el año 1967, Natentsun por lo menos ya aparecía en el Atlas arqueológico de la República mexicana (Piña, 1967: 53).

Sin embargo, los reclamos de Blom serían escuchados sólo varios años después por los arqueólogos Pierre Becquelin y Claude Baudez, de la Misión Arqueológica Francesa, quienes exploraron los tres sitios en 1977. Los arqueólogos franceses llevaron a cabo mediciones de todas las estructuras y elaboraron con ellas los croquis correspondientes. También rescataron diversos materiales arqueológicos, como restos de cerámica recolectados en superficie, y lograron datar con precisión los sitios en el Clásico Tardío. Todos estos resultados fueron publicados en su obra sobre Toniná, la cual incluyó un dibujo de la cabeza del muro de Natentsun, ilustración elaborada con base en las fotos de Duby, pues para cuando los estudiosos franceses trabajaron en el lugar ya había desaparecido esta obra de estuco (Becquelin y Baudez, 1982: 593-609, 1233-1239, 1242) (ver figura 1).

Años más tarde, en respuesta a una denuncia local por saqueo presentada ante el INAH, el arqueólogo Alejandro Tovalín Ahumada visitó los sitios en abril de 1993 con el objeto de llevar a cabo un diagnóstico de estos. El especialista efectuó un reconocimiento que incluía mediciones de todas las estructuras, y atestiguó el grave deterioro con respecto al estado reportado por los franceses, por lo cual hizo la denuncia correspondiente (Tovalín, 1993: 1-2). Los datos incluidos en ese reporte se publican por vez primera en el presente artículo. Desde la visita del arqueólogo, las autoridades mexicanas no han llevado a cabo ni impulsado trabajos de investigación o rescate en el lugar.

Afortunadamente, desde 1993 y hasta la fecha, el cronista de Chilón, el antropólogo Julio Alí Reyes Monterrosa, ha mantenido un constante seguimiento de estos sitios arqueológicos. En 1998, Reyes Monterrosa registró en fotografías el estado de conservación de las estructuras de Natentsun y de los otros sitios vecinos. También denunció ante distintas instancias el deterioro de los mismos (Reyes, 2009: 13-14). Por su parte, el geógrafo norteamericano Eric Samson, director del Proyecto Maya Esteem, realizó entre 1999 y 2007 varias visitas informales a Natentsun con el propósito de inspeccionar su estado y realizar estudios sobre el sitio, en las cuales evidenció nuevos pozos de saqueo (Samson, 1999-2007).

En marzo de 2010, niños de comunidades vecinas a este grupo de sitios arqueológicos informaron a sus profesores sobre el descubrimiento de más estructuras en las cercanías, en esta ocasión, al parecer intactas. Es difícil ingresar al lugar pues éste actualmente se encuentra en territorios ejidales. Sin embargo, Reyes Monterrosa (comunicación personal, 2010) ha logrado acercarse y fotografiar las nuevas estructuras. Desgraciadamente, día tras día estos monumentos son dañados por los propios ejidatarios en su constante intento por encontrar supuestos tesoros en las entrañas de los edificios.

\section{Las estructuras}

De acuerdo con lo registrado en la obra de Becquelin y Baudez (1982) y en el reporte inédito de Tovalín (1993), los cuatro sitios en cuestión presentan las siguientes características. El sitio de Chilón consiste sólo de una plataforma aislada sin cerámica de superficie. Mukana, por su parte, posee cuatro estructuras construidas sobre 
una meseta artificial alargada -90 por 25 metros-; en dos de esas estructuras se hallaron cuatro posibles tumbas abovedadas. Por el lado sur presenta al menos dos terrazas a menor nivel. No se halló material cerámico de superficie. El sitio de Nachoj, a su vez, consta de una estructura separada, llamada Nachoj A, $\mathrm{y}$ de tres pequeños montículos de menos de un metro de altura, elaborados sobre una amplia terraza (50 por 20 metros), denominados Nachoj B. En ninguno de los dos se halló cerámica de superficie (Becquelin y Baudez, 1982: 593-609, 1233-1239; Tovalín, 1993: 1-2).

Natentsun, por último, es un gran conjunto compuesto por seis estructuras de diferentes dimensiones y por una plaza central, todo levantado sobre la cima del cerro. En el borde norte de la plaza central se localizan seis tumbas abovedadas con nichos, todas ellas saqueadas. Cabe destacar que, al parecer, existía una tradición local de uso de criptas en terrazas, práctica que no se observa en otros sitios arqueológicos. En superficie se ha encontrado cerámica del período Posclásico Temprano (Becquelin y Baudez, 1982: 593609, 1233-1239; Tovalín, 1993: 1-2). Como se ha señalado, Natentsun es, de los tres sitios, el único que conservaba bellas figuras de estuco del periodo Clásico Tardío (ver foto 1 y figura 1), las que examinaremos más adelante.

En coherencia con el espíritu del presente trabajo, y para poder valorar las dimensiones del complejo arqueológico en cuestión, en la tabla l se presentan las medidas de cada una de las estructuras y tumbas de los sitios de acuerdo con datos proporcionados por Becquelin y Baudez (1982) y Tovalín (1993). ${ }^{2}$

\section{Los estucos}

En la figura fijada al muro, Cordan vio una variante de la cabeza del glifo para las unidades de tiempo llamadas k'atun en el sistema calendárico de los antiguos mayas (ver fotos 1 y 4, y figura 1), mientras que en la figura conservada en la casa de Jiménez observó la variante de la cabeza del glifo para las unidades bak'tun (ver foto 2) (Cordan, 1959: 70-113). Blom, quien sólo conoció la figura fijada al muro, señala sin embargo que el presunto glifo de k'atun no presenta numeral alguno, por lo que no podría corresponder al glifo de la unidad señalada; en su lugar, Blom ve un ejemplo del llamado Glifo F, el cual en las inscripciones calendáricas aparece precisamente sin numeral (Blom y Duby, 1959).

No obstante, las figuras, por sus rasgos, parecen corresponder, a nuestro parecer, a glifos de k'atun y bak'tun tal como lo propuso Cordan; los numerales, posiblemente elaborados también en su variante de cabeza, pudieron haberse destruido durante el saqueo. El glifo del bak'tun ${ }^{3}$ puede identificarse por el gran ojo y la mano puesta en la mandíbula del ser; por el contrario, el glifo del k'atun se puede reconocer por su ojo pequeño. Otros de los fragmentos de estuco muestran figuras antropomorfas, cráneos, posibles glifos y elementos que parecen corresponder a algunos de los distintivos del llamado Monstruo Witz, tales como sus características orejeras (ver foto 3). Recuérdese que imágenes de esta entidad decoraban precisamente los edificios de las ciudades del área central maya clásica. Sin embargo, resulta difícil dar una interpretación definitiva de todas estas obras debido al estado fragmentario en el que fueron halladas.

\section{Discusión}

A juzgar por las características del conjunto de monumentos y obras encontrado en estos cuatro sitios, entre ellos los bellos estucos modelados del periodo Clásico Tardío de Natentsun, es claro que este complejo arqueológico fue un centro de relevancia en la historia antigua de la comarca.

Relacionado con lo anterior, es de notar la presencia de otro sitio de importancia a solo dos kilómetros al norte en línea recta. Se trata del sitio arqueológico denominado Bolonkin, el cual destaca sobremanera por presentar estructuras arquitectónicas, tumbas, escultura monumental y portátil de piedra, así como restos de pinturas murales, datados todos para el lapso que va desde finales del siglo VI d. C. hasta finales del siglo VII, es decir, entre finales del Clásico Temprano y la primera parte del Clásico Tardío (Andrieu et al., 2012; Sheseña y Tovalín, en prensa) (ver mapa 1). 
Creemos que la ubicación de Bolonkin, y por ende del complejo arqueológico motivo del presente artículo, en un corredor natural útil para el comercio hacia Tabasco (Navarrete, 1973: 63), habría sido decisiva en el posicionamiento de todas estas entidades en la red política de la región (Sheseña y Lee, 2004: 130-131), incluyendo por supuesto a Chilón, Mukana, Natentsun y Nachoj. Pero también habría condicionado no sólo su atractivo para entidades vecinas más fuertes y agresivas, sino también su involucramiento en conflictos de grandes dimensiones, como el desarrollado entre Toniná y Palenque (Sheseña y Lee, 2004: 130-131). A partir de un yugo con jeroglíficos procedente de Toniná, pero hallado en Bolonkin, Alejandro Sheseña y el arqueólogo recientemente fallecido Thomas Lee (2004: 130-131) han propuesto justamente que Bolonkin, de algún modo, habría formado parte del área de influencia de Toniná en tiempos del gobernante K'ihnich Baaknal Chaak ${ }^{4}$ hacia finales del siglo VII d. C., sugerencia compartida por Chloe Andrieu y colegas (2012: 9) y por Eric Taladoire (2012). Es posible que Chilón, Mukana, Natentsun y Nachoj hayan formado parte de este mismo escenario.

Por otro lado, el estilo de los estucos modelados de Natentsun permite considerar el complejo arqueológico en cuestión como contemporáneo a las inscripciones del Clásico Tardío otrora pintadas en el interior de la cueva de Yaleltsemen, ubicada a siete kilómetros en línea recta al sur de Chilón, en las cercanías del actual poblado de Bachajón, precisamente detrás de Chilón, Mukana, Natentsun y Nachoj (ver mapa 1). Cabe destacar que una de las inscripciones de esta cueva hace referencia a la llegada a esta caverna de un personaje proveniente de cierta entidad política importante existente en ese entonces en la región y que a la fecha no ha sido localizada. Se trata del sitio denominado, según su glifoemblema, Sibikte' (sibik-te') (Stuart, en Bassie, 2002 y en Skidmore, 2004).

Otras referencias sobre esta entidad las encontramos en el Monumento 172 de Toniná (Clásico Tardío) (Skidmore, 2004) y en una inscripción pintada en otra cueva, la de Jolja, un lugar localizado esta vez al norte de Chilón, a 27 kilómetros en línea recta (Sheseña, 2007) (ver mapa 1). Se trata del llamado Grupo 6 de Jolja, el cual señala la llegada a esta cueva de un personaje también proveniente de Sibikte' el 26 de diciembre del año 528 d.C., fecha contemporánea a la de algunos de los murales de Bolonkin (Sheseña, 2007: 12-13; Andrieu et al., 2012; Sheseña y Tovalín, en prensa). Dato interesante es el hecho de que en las inscripciones de ambas cuevas se use el mismo verbo a-ni-ya, ahnii, (estuvo, llegó) (Sheseña, 2007), tal vez como reflejo de un estilo discursivo regional. Todas estas menciones muestran que Sibikte' fue un centro destacado en el antiguo orden político de la región de Chilón (Taladoire, 2012).

Todas estas evidencias hacen difícil no pensar en la posibilidad de algún tipo de relación entre Chilón, Mukana, Natentsun, Nachoj, Bolonkin y las cuevas de Yaleltsemen y Jolja. Ya anteriormente Sheseña había propuesto que la ciudad de Sibikte' podría haberse localizado en algún lugar en las inmediaciones de Bachajón (Sheseña, 2007: 18-19). En vista de la riqueza arqueológica, sin equivalentes hasta el momento en esta región, hallada en Bolonkin y en Natentsun -y en Chilón, Muktana y Nachoj-, los que suscriben el presente trabajo no descartan la posibilidad de que, concretamente en Bolonkin, o en el complejo arqueológico que nos atañe, se haya localizado el sitio perdido de Sibikte'. Futuros descubrimientos con seguridad ayudarán a confirmar o negar esta posibilidad.

\section{Notas}

${ }^{1}$ Alejandro Sheseña quisiera agradecer a Julio Alí Reyes Monterrosa y familia su invaluable labor en Chilón y el auxilio prestado durante todos estos años.

2 Para ver los croquis de estas tumbas y estructuras, consultar las obras referidas de Becquelin, Baudez y Tovalín.

${ }^{3}$ El glifo de bak'tun actualmente se lee pik (Stuart, 2006).

${ }^{4}$ El gobernante de Toniná K'ihnich Baaknal Chaak reinó en el lapso de 688-704? d.C. según los estudios de Martin y Grube (2007: 180-183). 


\section{Referencias bibliográficas}

Andrieu, Chloe, Christophe Helmke, Harri Kettunen, Eric Taladoire y Robert Tamba (2012), "Maya Mural Paintings of Bolonkin, Chiapas, Mexico", en Mexicon. Aktualle Informationen und Studieszu Mesoamerika, vol. XXXIV, núm. l, febrero, pp. 7-10.

Bassie-Sweet, Karen (2002), Jolja Cave Project. FAMSI Final Report (manuscrito inédito).

Becquelin, Pierre y Claude F. Baudez (1982), Tonina: Une cité maya du Chiapas (Mexique), 3 t., México: Centre d’Etudes Mexicaines et Centraméricaines.

Blom, Frans (1961), "Notas sobre algunas ruinas todavía para explorar", en VIII Mesa Redonda: Los mayas del sur y sus relaciones con los nahuas meridionales, México: Sociedad Mexicana de Antropología, pp. $115-125$.

Blom, Frans y Gertrude Duby (1958), Las ruinas de Natentzum, Muctana y otras cerca de Chilón, Chiapas, octubre de 1958. Para la Dirección de Monumentos Arqueológicos del INAH (manuscrito inédito conservado en la biblioteca del Museo Na Bolom en San Cristóbal de Las Casas).

Cordan, Wolfgang (1959), Geheimnisim Urwald. Entdeckungsfahrten auf den Spuren der Mayas, Berlín, Darmstadt y Viena: Deutsche Buch/ Gemeinschaft.

Martin, Simon y Nikolai Grube (2007), Chronicle of the Maya Kings and Queens, Londres: Thames and Hudson.

Navarrete, Carlos (1973), "El sistema prehispánico de comunicaciones entre Chiapas y Tabasco", en Anales de Antropología, vol. X, México: UNAM, pp. 33-92.

Piña Chan, Román (1967), Atlas arqueológico de la República mexicana, México: Instituto Nacional de Antropología e Historia.
Reyes Monterrosa, Julio Alí (2009), Chilum: geografía, historia y tradición de la "Tierra Dulce", San Cristóbal de Las Casas: Philadelphia.

Riese, Berthold (2004), "Abkürzungenfür MayaruinenortemitInschriften”, en Wayeb Notes, núm. 8. 〈http://www.wayeb.org/notes/wayeb_notes0008. pdf $>$ [2 de abril de 2013].

Samson, Eric (1999-2007), Mayan Esteem Project/Proyecto Estima de Maya. 〈http://www.mayanesteem.org〉 [2 de abril de 2013].

Sheseña, Alejandro (2007), "Los textos jeroglíficos mayas de la cueva de Jolja, Chiapas", en Mesoweb, 〈www.mesoweb.com/es/articulos/jolja/Jolja.pdf〉 [2 de abril de 2013].

Sheseña, Alejandro y Thomas A. Lee Whiting (2004), "Yugo incrustado con glifos mayas procedente de los alrededores de Chilón, Chiapas", en Mexicon. Aktualle Informationen und Studies zu Mesoamerika, vol. XXVI, núm. 6, diciembre, pp. 127-132.

Sheseña, Alejandro y Alejandro Tovalín (en prensa), "Las pinturas mayas del sitio de Bolonkin, Chiapas". Skidmore, Joel (2004), "New Ballplayer Panel from Tonina”, en Mesoweb, 〈http://www.mesoweb.com/ reports/tonina_Ml72.html> [2 de abril de 2013].

Stuart, David (2006), "Jade and Chocolate: Bundles of Wealth in Classic Maya Economics and Ritual", en Julia Guernsey y F. Kent Reilly (eds.), Sacred Bundles. Ritual Acts of Wrapping and Binding in Mesoamerica, Bernardsville: Boundary end Archaeology Research Center, pp. 127-144.

Taladoire, Eric (2012), Towards a Reevaluation of the Tonina Polity (manuscrito inédito).

Tovalín Ahumada, Alejandro (1993), Informe de la visita a los sitios de Naxtenxum, Nachoj y Mukana, Municipio de Chilón, Chiapas: Centro Regional del INAH en Chiapas (manuscrito inédito). 


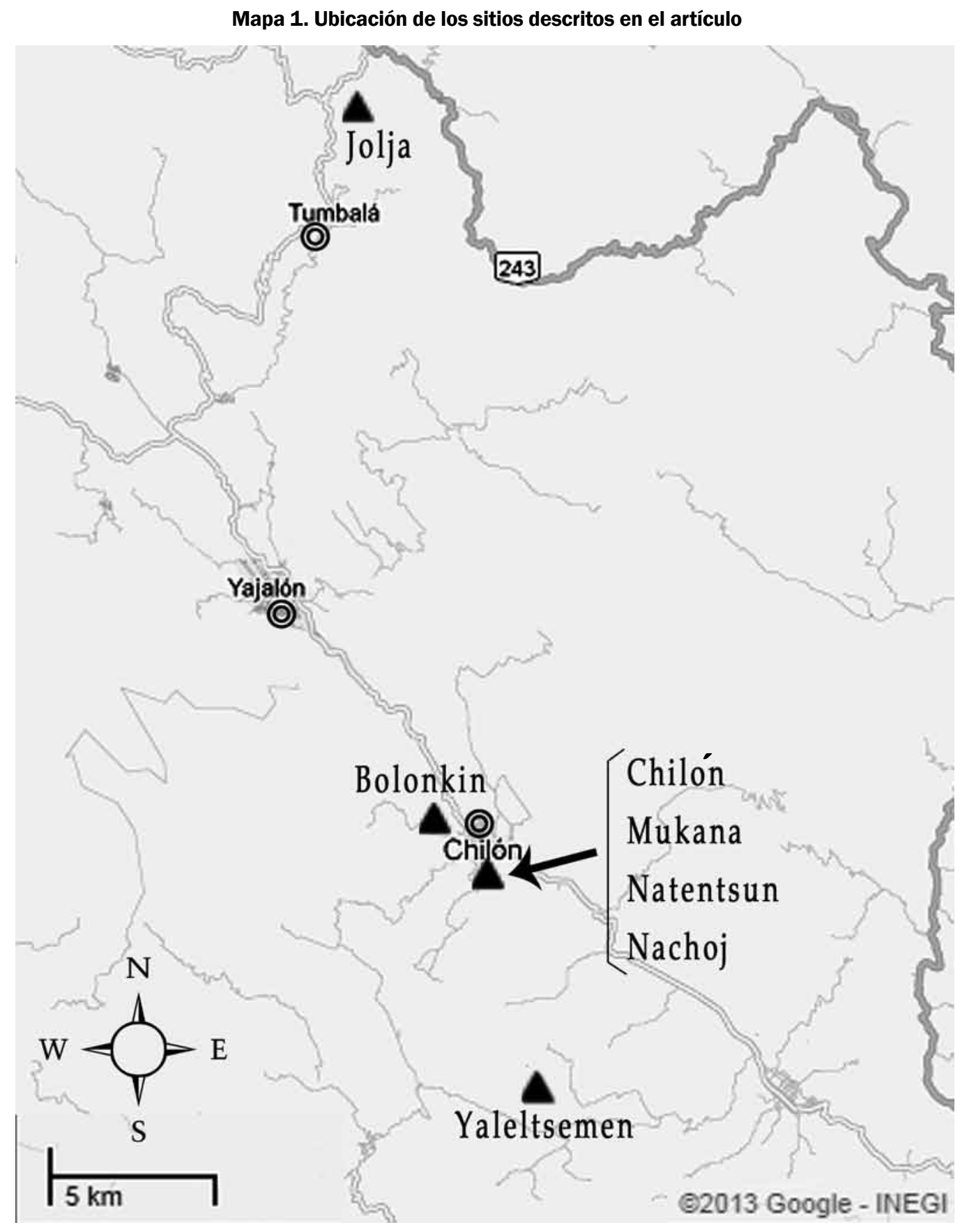

Fuente: INEGI, 2013. 
Foto 1. Cabeza de estuco al descubierto en el sitio de Natentsun, año 1958

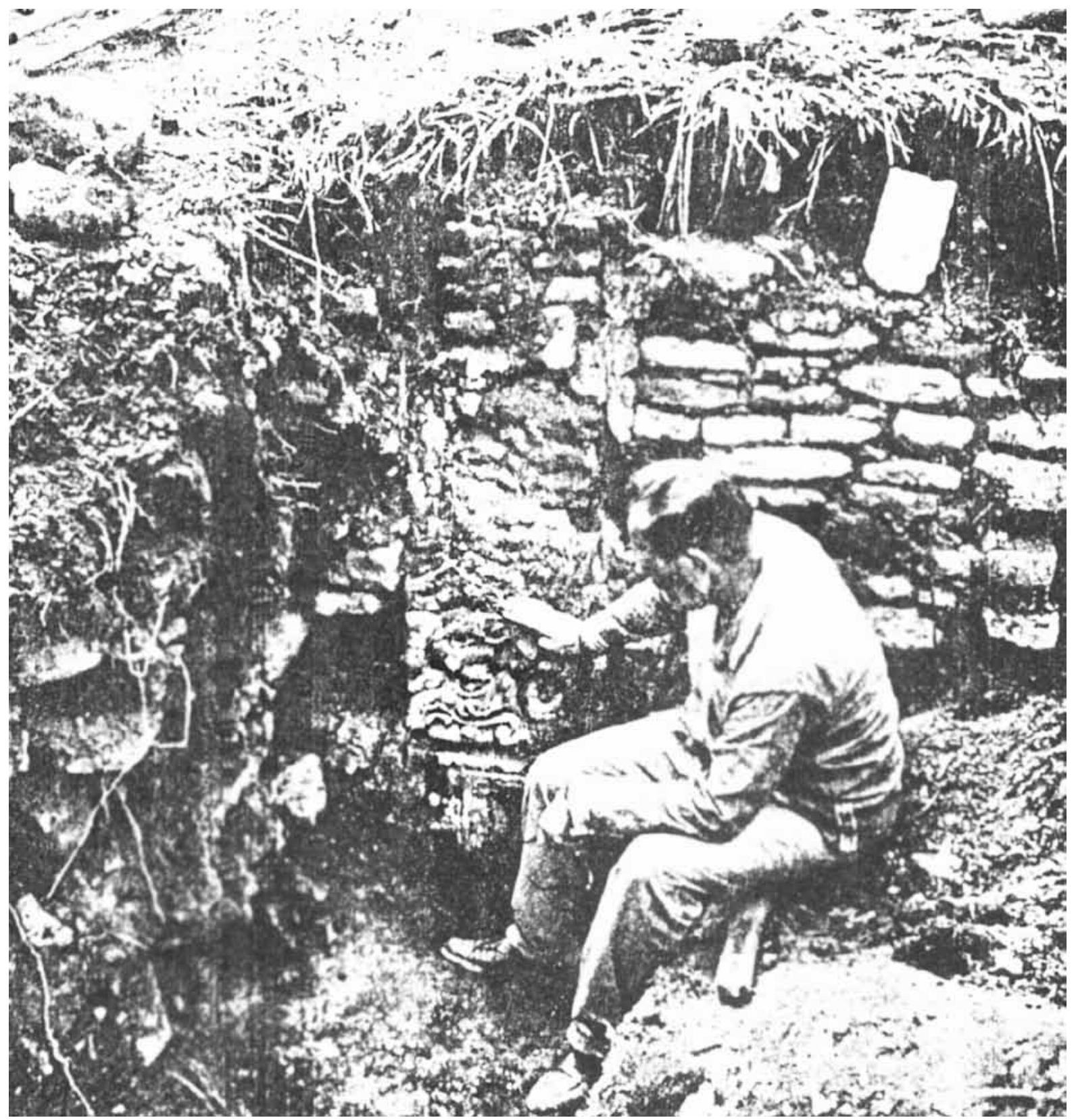

Fuente: Cordan, 1959. 
Foto 2. Otra cabeza de estuco proveniente de Natentsun, año 1958

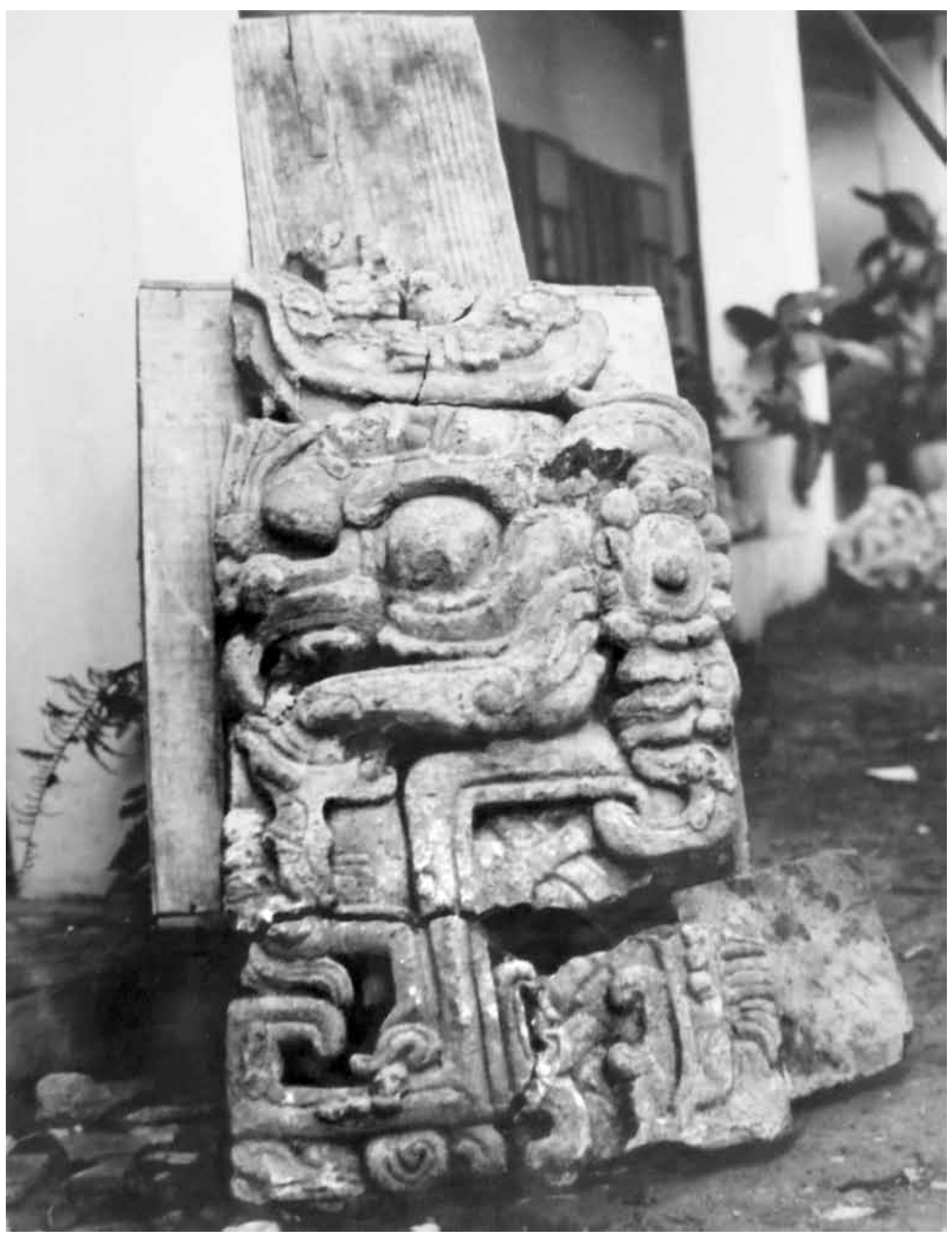

Fuente: Walter Sellschopp, 1958. 
Foto 3. Otros fragmentos de figuras de estuco extraídas de Natentsun, año 1958

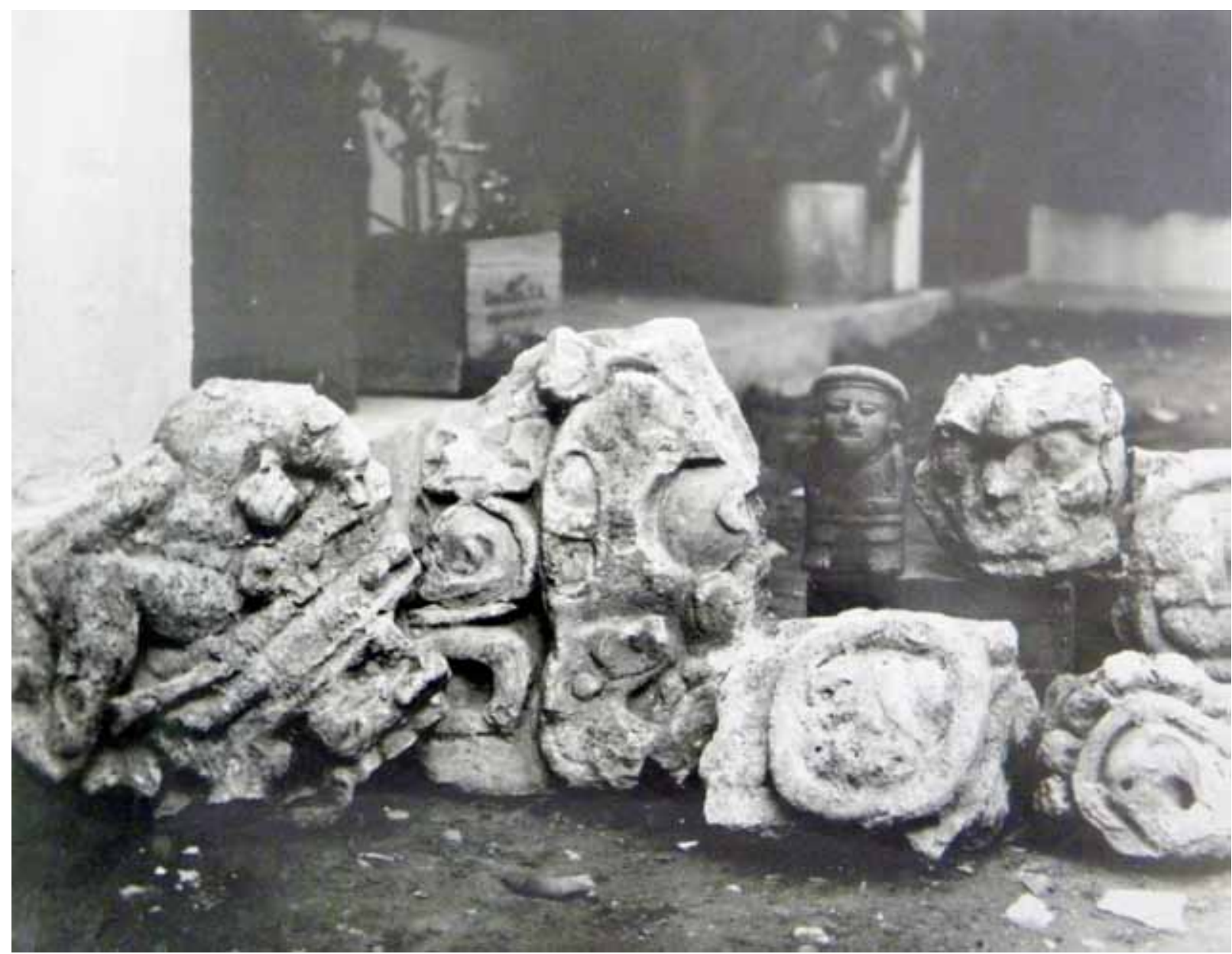

Fuente: Walter Sellschopp, 1958. 


\section{Foto 4. La cabeza de estuco de la figura 1 fotografiada por Gertrude Duby en 1958}

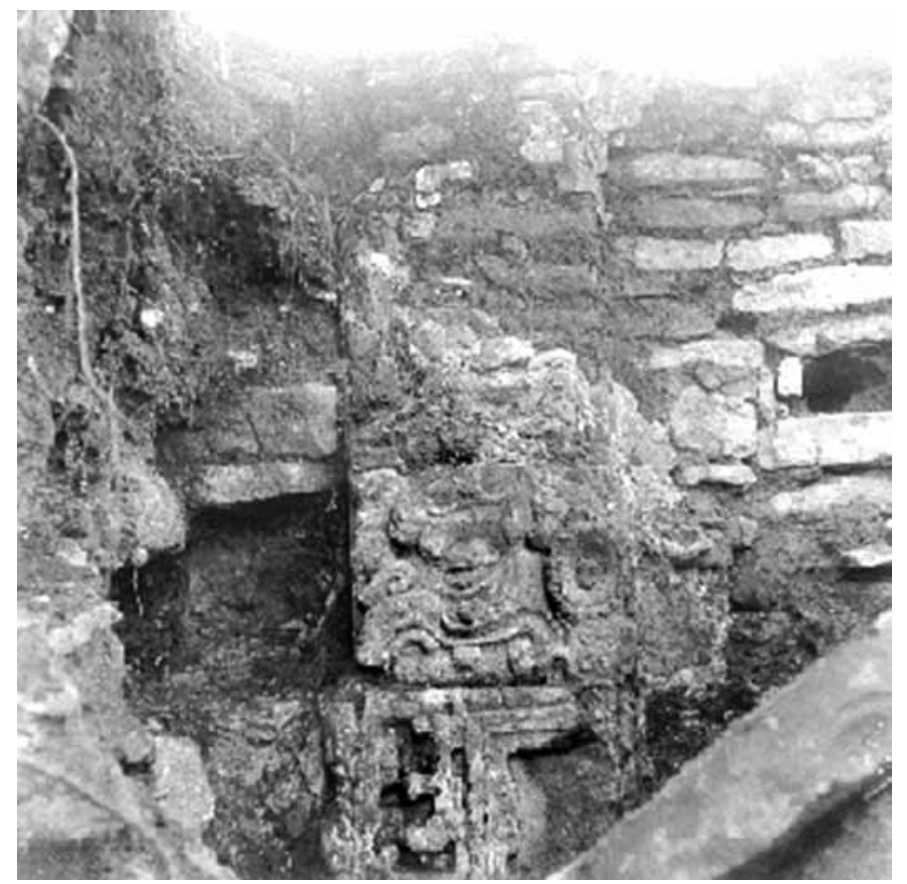

Fuente: Gertrude Duby, 1958.

\section{Figura 1. Dibujo de la cabeza de estuco de la foto 1}

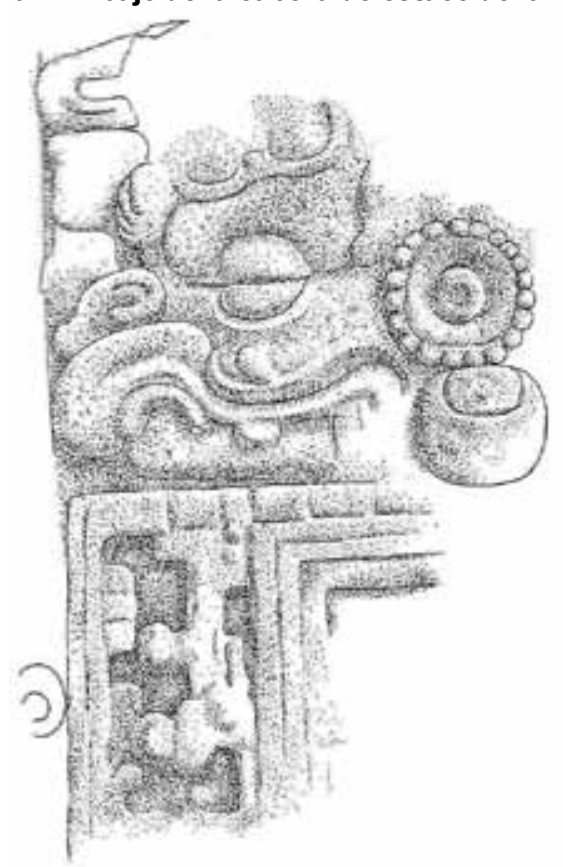

Fuente: Becquelin y Baudez (1982: 1242), hecho a partir de la foto de Duby de 1958 (foto 4). 
Tabla 1. Recopilación de las medidas de las estructuras de los cuatro sitios

\begin{tabular}{|c|c|c|c|c|c|}
\hline Sitio & Estructura & Tumba & Ancho & Largo & Alto \\
\hline Chilón & 1 & $x$ & $8.80 \mathrm{~m}$ & $14.50 \mathrm{~m}$ & $0.20 \mathrm{~m}$ \\
\hline \multirow{4}{*}{ Mukana } & 1 & \multirow{4}{*}{$x$} & $7.50 \mathrm{~m}$ & $7.50 \mathrm{~m}$ & $2 \mathrm{~m}$ \\
\hline & 2 & & $10 \mathrm{~m}$ & $17 \mathrm{~m}$ & $4 \mathrm{~m}$ \\
\hline & 3 & & $6 \mathrm{~m}$ & $30 \mathrm{~m}$ & $2 \mathrm{~m}$ \\
\hline & 4 & & $10 \mathrm{~m}$ & $15 \mathrm{~m}$ & $5 \mathrm{~m}$ \\
\hline \multirow{9}{*}{ Natentsun } & 1 & \multirow{6}{*}{$x$} & $6 \mathrm{~m}$ & $20 \mathrm{~m}$ & $1.20 \mathrm{~m}$ \\
\hline & 2 & & $20 \mathrm{~m}$ & $20 \mathrm{~m}$ & $1.50 \mathrm{~m}$ \\
\hline & 3 & & $7 \mathrm{~m}$ & $9 \mathrm{~m}$ & $0.80 \mathrm{~m}$ \\
\hline & 4 & & $12 \mathrm{~m}$ & $16 \mathrm{~m}$ & $1.70 \mathrm{~m}$ \\
\hline & 5 & & $2 \mathrm{~m}$ & $10 \mathrm{~m}$ & $3.40 \mathrm{~m}$ \\
\hline & 6 & & $7 \mathrm{~m}$ & $9 \mathrm{~m}$ & $0.30 \mathrm{~m}$ \\
\hline & \multirow{3}{*}{ Plaza central } & Tumba 1 & $0.79 \mathrm{~m}$ & $2.90 \mathrm{~m}$ & $0.90 \mathrm{~m}$ \\
\hline & & Tumba 5 & $0.60 \mathrm{~m}$ & $1.70 \mathrm{~m}$ & $0.59 \mathrm{~m}$ \\
\hline & & Tumba 6 & $0.60 \mathrm{~m}$ & $2.77 \mathrm{~m}$ & $0.90 \mathrm{~m}$ \\
\hline Nachoj A & 1 & $x$ & $11 \mathrm{~m}$ & $13 \mathrm{~m}$ & $2 \mathrm{~m}$ \\
\hline \multirow[t]{3}{*}{ Nachoj B } & 1 & \multirow{3}{*}{$x$} & $7.50 \mathrm{~m}$ & $10.50 \mathrm{~m}$ & $0.75 \mathrm{~m}$ \\
\hline & 2 & & $10 \mathrm{~m}$ & $11 \mathrm{~m}$ & $1 \mathrm{~m}$ \\
\hline & 3 & & $6 \mathrm{~m}$ & $8.30 \mathrm{~m}$ & $0.50 \mathrm{~m}$ \\
\hline
\end{tabular}

Fuente: datos tomados de Becquelin y Baudez (1982) y Tovalín (1993). 